\title{
Triaxiality of heavy nuclei as essential feature to predict radiative capture
}

\author{
E. Grosse ${ }^{1, \text { a }}$, A.R. Junghans ${ }^{2}$, and R. Massarczyk ${ }^{1,2}$ \\ ${ }^{1}$ Institute of Nuclear and Particle Physics, Technische Universität Dresden, 01062 Dresden, Germany \\ ${ }^{2}$ Institute of Radiation Physics, Helmholtz-Zentrum Dresden-Rossendorf, 01314 Dresden, Germany
}

\begin{abstract}
Cross sections for neutron capture in the range of unresolved resonances and average level distances near the neutron emission threshold are simultaneously predicted for more than 140 spin- 0 target nuclei with $\mathrm{A}>50$. Assuming triaxiality in nearly all these nuclei a combined description of both, photon strength and level density, is presented - needing very few fit parameters only.
\end{abstract}

\section{Introduction}

The radiative capture of neutrons in the $\mathrm{keV}$ to $\mathrm{MeV}$ range by heavy nuclei plays an important role in considerations for advanced systems aiming for a reduction of radioactive nuclear waste [1]. This process is of interest also for the cosmic nucleosynthesis, especially for scenarios with neutron capture leading to a production of nuclides beyond $\mathrm{Fe}$ by the s-process [2]. Usually predictions for radiative neutron capture cross sections in the range of unresolved resonances are based on statistical model calculations. Their reliability depends not only on the proper characterization of the input channel, but more strongly on the details determining the decay of the intermediately formed compound nucleus. Here the strength of its electromagnetic decay is of importance as well as the open phase space in the final nucleus, i.e. the density of levels reached by the first photon emitted. As both quantities have to be known also for unstable isotopes we present a parameterization for the capture by even-even nuclei, which is global and thus expected to be applicable also away from stability.

\section{Dipole strength functions.}

The electric dipole strength functions used are derived from a global fit to IVGDR shapes by the sum of three Lorentzians (TLO), adding up to the TRK sum rule and based on theoretical predictions for the A-dependence of pole energies and spreading widths [3]. As in a first step we only consider s-capture on $J=0$ targets we need to show that our ansatz also works for odd nuclei (cf. Fig.1); we use average information on $\beta$ and $\gamma$ from an CHFB calculation [4] for even neighbour nuclei. Data on $f_{1}$ are from neutron and photon induced processes.

\footnotetext{
a Corresponding author: E.Grosse@tu-dresden.de
}

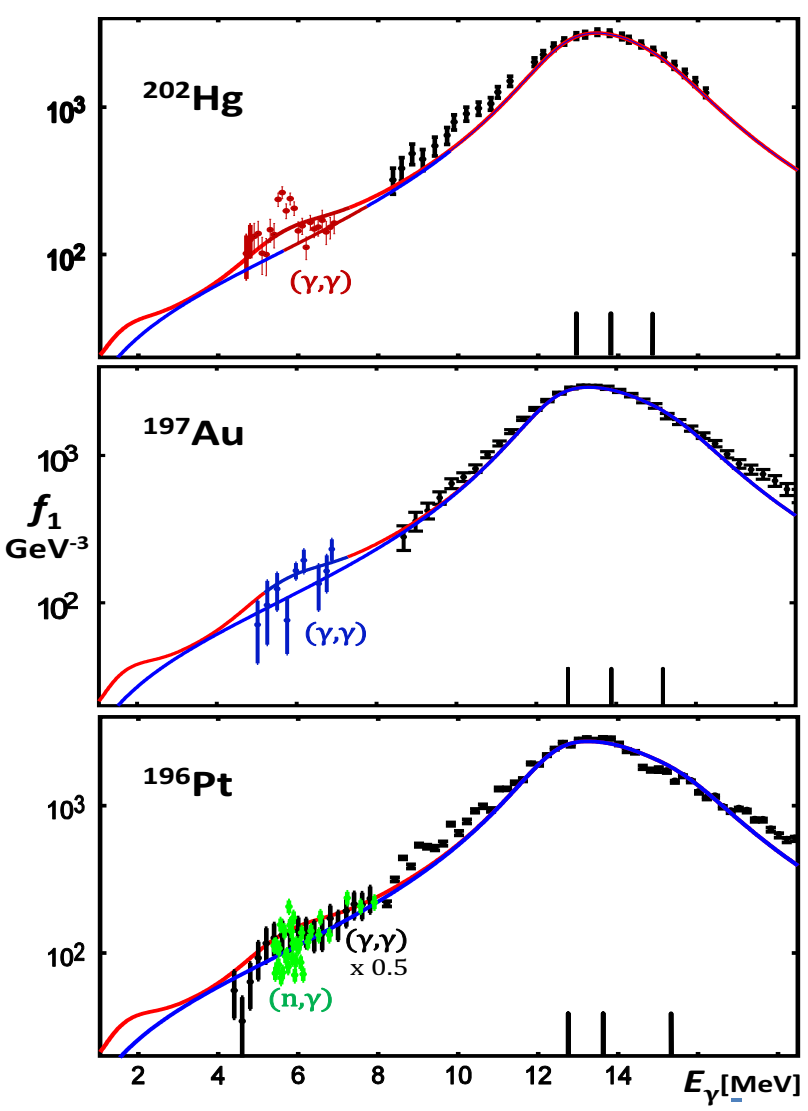

Figure 1. Prediction for dipole strength functions in $\mathrm{Hg}$ (top), ${ }^{197} \mathrm{Au}$ (middle) and ${ }^{196} \mathrm{Pt}$ (bottom) compared to data from $(\gamma, \mathrm{n})$ experiments ( black) [5] and photon emission ( + red, blue, green or black) [6]; the factor 0.5 indicates the strongest possible reduction due to variations in the level density. A blue line presents the E1-strength predicted by TLO with the poles indicated as bars; the inclusion of minor strength is depicted as full red line. 


\section{Level density in nuclei with broken axial symmetry}

For the case of even-even target nuclei standard Fermi gas formulae [7], modified to incorporate triaxiality [8], describe average neutron capture resonance distances well. The dependence of the quasi-particle (qp) state density on the excitation energy $E_{\mathrm{x}}$ is assumed to be exponential $[7,8]$ in the pairing dominated phase below a critical temperature $t_{\mathrm{c}}[10-12]$. In contrast to previous work [7, 11] we fix the transition point on the basis of Fermi gas theory with the energy corrected by a back shift [10]. For it the sum of the condensation energy [1013] and a shell correction is used. The latter is taken from a comparison of nuclear masses to liquid drop values, and the rather strong effect of it is assumed to be negligible in view of the near identity of the one used by us [15] as compared to a previous fit [16]. Only one free global fit parameter, a surprisingly small surface term quantifying the difference of the level density parameter a from the value predicted for nuclear matter $\mathrm{a}_{\mathrm{nm}} \approx \mathrm{A} / 15$, is needed to obtain agreement to data on an absolute scale.

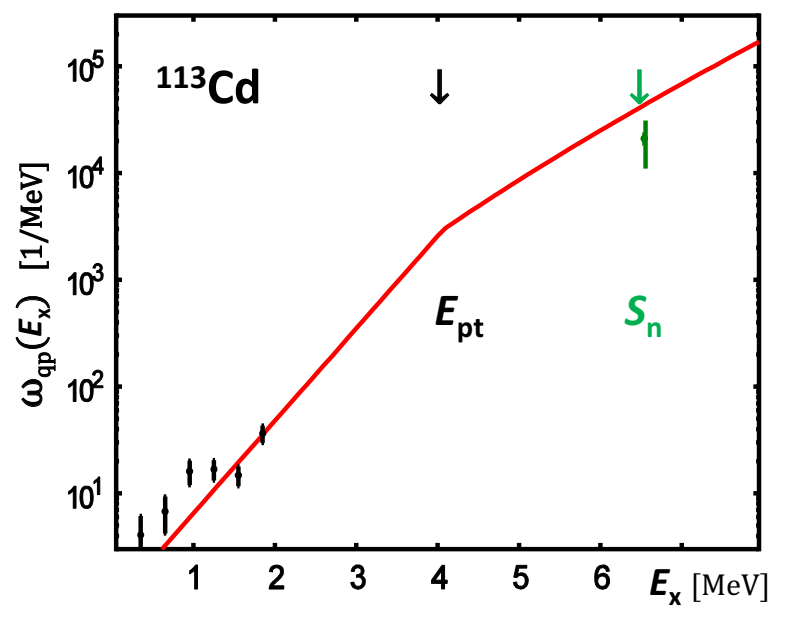

Figure 2. For the state density $\omega_{\mathrm{qp}}\left(E_{\mathrm{x}}\right)$ in ${ }^{113} \mathrm{Cd}$ the prediction is shown together with respective data [11] obtained from the level scheme (black) and resonance spacings (green). A change in slope at the phase transition energy $E_{\mathrm{pt}}$ is clearly seen.

The absolute scale of our prediction for the level density is strongly influenced by allowing for broken axial shape symmetry in the Fermi gas regime. Using the formulae for the projection of quasi-particle states in a nucleus without axial symmetry [9] we arrive at level densities $\rho\left(J, E_{\mathrm{x}}\right)$ in the observer's system, which are increased by about one order of magnitude as compared to the case with only axial deformation. In previous work [8, 11-13] this may have been compensated by an increase of the level density parameter ã, eventually adjusted in a fit. We do without such a modification to not induce a change in the excitation energy dependence of $\rho$ at variance to the Fermi gas, which we accept as proper description of the statistics in highly excited nuclei.

To demonstrate the dependence of our ansatz on the nuclear mass number A the comparison to the spacings between resonances populated in s-capture of neutrons by 146 even-even nuclei is shown in Fig. 3. Apparently the respective experimental information, recently updated within RIPL-3 [11], was never compared to a calculation not assuming at least axial symmetry. In some previous work $[8,11,12]$ even spherical symmetry was assumed without rotational collective enhancement. Significant modifications on ã generated a sufficiently large $\omega_{\mathrm{qp}}$ and up to $\tilde{a} \approx \mathrm{A} / 9$ was needed [12], although an enhancement by vibrational collectivity was introduced. In agreement to our findings this increases $\rho$ by less than a factor of 1.5, which would hardly be seen in Fig.3, depicting our results for resonance spacings just above the neutron binding energy $\mathrm{S}_{\mathrm{n}}$. The drawn curve represents an average $\tilde{\mathrm{a}} \approx \mathrm{A} / 13$ as it includes the surface correction term $\mathrm{A}^{2 / 3} / 17$. We find good agreement to the data over the full mass range, which depends only marginally on the damping of the shell effect, which was included without the introduction of an additional parameter [14].

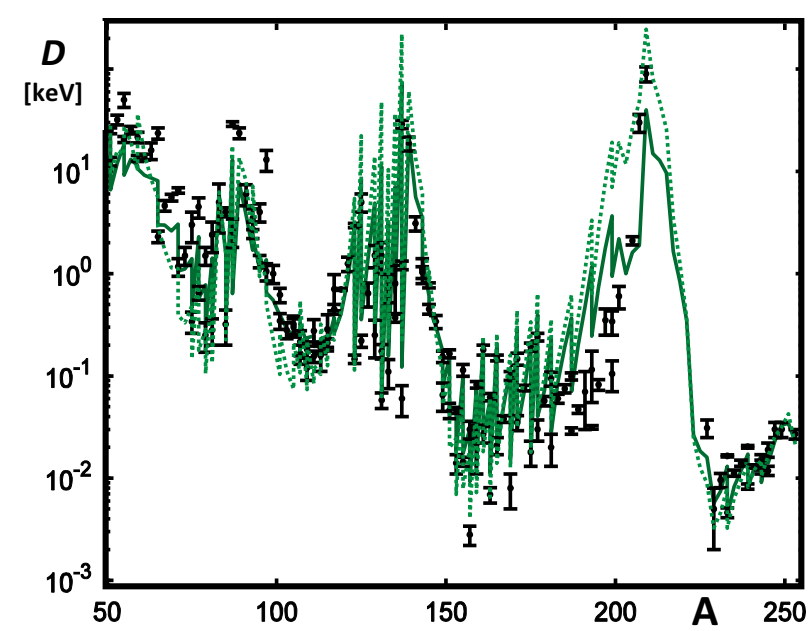

Figure 3. Experimental information [11] on average resonance spacings near $S_{\mathrm{n}}$ (black $\boldsymbol{\Phi}$ ) versus nuclear mass A. The prediction shown as drawn curve was obtained including a shell correction [15], damped with $E_{x}[14]$; the dots depict the no-damping case.

To obtain the good agreement depicted in Fig.3 for resonance spacings a significant increase of the level density in comparison to the quasi-particle value was needed. As discussed above, a deviation from symmetry results in additional degrees of freedom and a collective enhancement. We find, that the breaking of spherical and axial symmetry leads to an increase by at least $\mathrm{A} / 2$, even if the triaxiality is small $[4,9]$. The predicted additional increase caused by axial symmetry breaking was never regarded previously $[7,8,11-14]$ and the procedure used for the treatment of rotational enhancement [11] finds a level density increase as compared to sphericity, which is a factor 4-9 below the value we find.

\section{Radiative neutron capture}

The effect of a combination of our ansatz on the photon strength and for the level density - both allowing for a breaking of axial symmetry - can be well demonstrated on absolute scale by the comparison of predicted to measured radiative neutron capture cross sections. For the study of their dependence on the nuclear mass A we take advantage of a recent compilation [2] of respective 
measurements and their inter- and extra-polation to a Maxwellian distribution centred around an energy corresponding to a temperature $T_{\mathrm{AGB}}=30 \mathrm{keV}$. Maxwellian average cross sections [2] around $30 \mathrm{keV}$ are characteristic for the interior of red giant stars, where in the s-process stellar nucleosynthesis takes place. At this low energy only s-capture has to be considered and a quasi-classical treatment [17] of the reaction dynamics is adequate. On the other hand the neutron width already exceeds the average photon width and the cross section factorizes in a level density and a photon strength component [17]. As becomes obvious in Fig. 4, our parameterization of these ingredients allows a good prediction on an absolute scale over many orders of magnitude, very surprising in view of the few adjusted quantities. The dotted line depicts the E1-contribution as parameterized by our TLO-ansatz [3], the drawn curve demonstrates the additional contribution due to three minor strength components [6] of some importance for radiative neutron capture:

1. Pygmy dipole strength of probably isoscalar nature,

2. E1 strength due to the coupling of $2^{+}$and $3^{-}$-vibrations,

3. orbital magnetic dipole strength related to deformation.

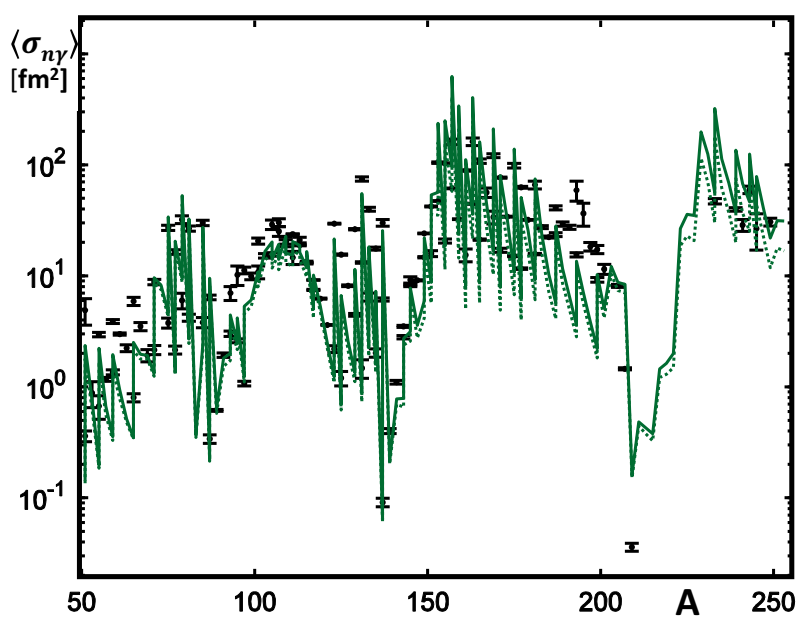

Figure 4. Comparison of predicted neutron capture cross sections $\langle\sigma(\mathrm{n}, \gamma)\rangle_{r}$ (full red curve, $\ell=0$ ) to experimental data on Maxwellian averaged cross sections [2] for $k T_{\mathrm{AGB}}=30 \mathrm{keV}$ vs. A. The dotted curve was calculated with TLO only.

\section{Conclusions}

Various spectroscopic data indicate triaxiality for a number of heavy nuclei $[3,4]$; two effects - hitherto not emphasised as such - indicate a breaking of axial symmetry:

1) With one global parameter the scheme proposed here reproduces observations for level densities in nuclei with $\mathrm{A}>50$ and $J=1 / 2$, when (a) the condensation energy $E_{\text {con }}$ is included in the Fermi gas backshift and (b) the collective enhancement due to symmetry reduction by triaxiality is included. This is achieved although the level density parameter ã has to be modified little from its nuclear matter value to fit resonance spacing data; we use a small surface term and the change is smaller than usual $[8,11,12]$.
2) Again only one global parameter suffices to fit to the shape of the IVGDR peak by a triple Lorentzian photon strength (TLO) - considerably improved and in accord to the TRK sum rule. It also predicts its low energy tail - without other modification than the addition of minor modes - to match respective strength data as well as neutron capture cross sections taken in the energy range of unresolved resonances.

For the last-mentioned finding a combination of the points 1) and 2) is needed, which is easily performed by considering spherical and axial symmetry to be broken as shown for low excitation by HFB calculations [4] and as expected to increase with energy. Exact deformation parameters are unimportant for the tail of the E1resonance as well as for the density of low spin states occurring in neutron capture by even targets as neither spin cut off nor moments of inertia are involved. At variance to previous work [e.g. 10] the breaking of axial symmetry in excited heavy nuclei is demonstrated here on the basis of experimental data: For more than 140 spin-0 target nuclei with $\mathrm{A}>50$ level distance data and average capture cross sections are well described by a global ansatz. It promises reliable predictions for compound nuclear reactions also outside the valley of stability - as important for nuclear astrophysics and for the transmutation of nuclear waste.

\section{References}

1. M. Salvatores and G. Palmiotti, Prog. Part. Nucl. Phys. 66, 144 (2011)

2. I. Dillmann et al., Phys. Rev. C 81, 015801 (2010); id., //www.kadonis.org

3. A.R. Junghans et al., Phys. Lett. B 670, 200 (2008)

4. J.-P. Delaroche et al., Phys. Rev. C 81 (2010) 014303, incl. supplemental material

5. B.L. Berman and S.C. Fultz, Rev. Mod. Phys. 47, 713 (1975); cf. nndc.bnl.gov/exfor/endf00

6. R. Massarczyk et al., Phys. Rev. C 044306 (2013); id., Phys. Rev. Lett. 112, 072501 (2014)

7. A. Gilbert and A.G.W. Cameron, Canadian Journal of Physics 43, 1446 (1965)

8. T. v. Egidy and D. Bucurescu, Phys. Rev. C 72, 044311 (2005)

9. S. Bjørnholm, A. Bohr and B. Mottelson, IAEA conf. on fission, held at Rochester 1973; Report IAEA-STI/PUB/347, 367 (1974)

10. M.K. Grossjean and H. Feldmeier, Nucl. Phys. A 444, 113 (1985)

11. R. Capote et al., Nuclear Data Sheets 110, 3107 (2009); id., //www-nds.iaea.org/RIPL-3

12. A.V. Ignatyuk et al., Phys. Rev. C 47, 1504 (1993)

13. A.R. Junghans et al., Nucl. Phys. A 629, 635 (1998)

14. S.K. Kataria, V. S. Ramamurthy, and S. S. Kapoor, Phys. Rev. C 18, 549 (1978)

15. J.M. Pearson, Hyp. Int 132, 59 (2001)

16. W.D. Myers and W.J. Swiatecki, Ark. Fizik 36, 343 (1967)

17. A. M. Lane and J. E. Lynn, Proc. Phys. Soc. (London) A70, 557 (1957) 
